ACCEPTED MANUSCRIPT

\title{
The discretized Boussinesq equation and its conditional symmetry reduction
}

To cite this article before publication: Decio Levi et al $2019 \mathrm{~J}$. Phys. A: Math. Theor. in press https://doi.org/10.1088/1751-8121/ab5b47

\section{Manuscript version: Accepted Manuscript}

Accepted Manuscript is "the version of the article accepted for publication including all changes made as a result of the peer review process, and which may also include the addition to the article by IOP Publishing of a header, an article ID, a cover sheet and/or an 'Accepted Manuscript' watermark, but excluding any other editing, typesetting or other changes made by IOP Publishing and/or its licensors"

This Accepted Manuscript is @ 2019 IOP Publishing Ltd.

During the embargo period (the 12 month period from the publication of the Version of Record of this article), the Accepted Manuscript is fully protected by copyright and cannot be reused or reposted elsewhere.

As the Version of Record of this article is going to be / has been published on a subscription basis, this Accepted Manuscript is available for reuse under a CC BY-NC-ND 3.0 licence after the 12 month embargo period.

After the embargo period, everyone is permitted to use copy and redistribute this article for non-commercial purposes only, provided that they adhere to all the terms of the licence https://creativecommons.org/licences/by-nc-nd/3.0

Although reasonable endeavours have been taken to obtain all necessary permissions from third parties to include their copyrighted content within this article, their full citation and copyright line may not be present in this Accepted Manuscript version. Before using any content from this article, please refer to the Version of Record on IOPscience once published for full citation and copyright details, as permissions will likely be required. All third party content is fully copyright protected, unless specifically stated otherwise in the figure caption in the Version of Record.

View the article online for updates and enhancements. 


\section{Introduction}

Abstract. In this article we show that we can carry out the symmetry preserving discretization of the Boussinesq equation with respect to three of its more significant conditional symmetries. We perform the symmetry reduction of the obtained nonlinear discrete schemes with respect to the conditional symmetries and obtain the reduced discrete equations which unlike in the continuous case, are not always reducible to second order difference equations. A numerical comparison with the exact continuous solution given by Weierstrass elliptic functions is carried out.

Nonlinear Partial Differential Equations,(PDEs), which are found everywhere in modern physics [47], are often difficult to solve. One of the approaches to find analytical solutions is the symmetry reduction method based on the work by Sophus Lie and described in many books, see for example $[6,42]$. Not all PDEs have Lie point symmetries, and many times the symmetries do not provide the type of solutions we are looking for. So one looks for extensions or modifications of the construction which could overcome some of these problems. A technique not satisfying all the properties of a Lie group but just providing solutions by symmetry reduction was introduced by Bluman and Cole with the name non-classical method [5]. It consists of adding a well defined "condition" to the set of determining equation. Conditional symmetries transform a subset of solutions into solutions. They do not form a group but they can be used to obtain additional reductions leading to explicit solutions. Conditional symmetries and the reductions provided by them have been applied to obtain explicit (new, physically relevant) solutions for problems arising in non-newtonian fluid flow models [1], in magnetohydrodynamics [20], in reaction-diffusion-convection equations [12], in equations of heat and acoustics [18], etc. .

This research is part of a general program the aim of which is to make full use of the theory of Lie group to study the solution space of discrete equations and in particular to solve difference equations $[21,24,25,27,29,30,34-36]$. One of the questions 


\section{The discretized Boussinesq equation and its conditional symmetry reduction}

is how to do the symmetry preserving discretization, i.e. how to construct discretized equations which possess the same symmetries or a subgroup of the symmetry group of a given PDE $[2-4,7,10,11,15-17,24-26,43-45,49]$. The construction of the Partial Difference Equation ( $\mathrm{P} \Delta \mathrm{E})$ is obtained through the computation of discrete invariants of the symmetry group of the PDE or one of its subgroups looking for a function of these invariants which yields the PDE in the continuous limit.

In this article we will be interested in showing that we can discretize the Boussinesq equation preserving the conditional symmetries.

In Section 2 we recall the construction of the Boussinesq equation starting from the conditional symmetries and its symmetry reductions while in Section 3 we provide the conditionally invariant discretizations of the Boussinesq equation together with its invariant lattices, i.e. the discrete Boussinesq scheme. Section 4 is devoted to carrying out the reduction of the so obtained discretized Boussinesq schemes to ordinary difference schemes as well as a numerical comparison with/the exact result of the continuous case. In Section 5 we present a summary of the results, some concluding remarks and prospects for future works.

\section{Construction of nonlinear PDEs with conditional symmetries: the conditional symmetries of the Boussinesq equation}

Consider a $\operatorname{PDE} \mathcal{E}=0$ in $\mathbb{R}^{n}$ that is invariant under a continuous group of Lie point transformations. The corresponding infinitesimal symmetry generator is given as

$$
\hat{X}=\boldsymbol{\xi}(\mathbf{x}, u) \cdot \nabla+\phi(\mathbf{x}, u) \partial_{u},
$$

To construct conditional symmetries using the non-classical method one adds an auxiliary first-order equation to $\mathcal{E}=0$, build up in terms of the coefficients of the infinitesimal generator $\hat{X}$, namely

$$
\mathcal{C}=\mathcal{C}(\mathbf{x}, u, \nabla u)=\boldsymbol{\xi}(\mathbf{x}, u) \cdot \nabla u-\phi(\mathbf{x}, u)=0,
$$

the infinitesimal symmetry generator (1) written in characteristic form [42] set equal to zero. Equation (2) will be determined together with the vector field $\hat{X}$, as it involves the same functions $\boldsymbol{\xi}$ and $\phi$. In fact we look for the simultaneous symmetry group of the overdetermined system of equations $\mathcal{E}=0$ and $\mathcal{C}=0$. It is easy to prove that $(2)$ is weakly invariant under the first prolongation of $(1)$

$$
\operatorname{pr} \hat{X} \mathcal{C}=-\left(\boldsymbol{\xi}_{u} \cdot \nabla u-\phi_{u}\right) \mathcal{C}
$$

without imposing any conditions on the functions $\boldsymbol{\xi}$ and $\phi$. Consequently, we need just to apply the following invariance condition

$$
\left.\operatorname{pr} \hat{X} \mathcal{E}\right|_{(\mathcal{E}=0, \mathcal{C}=0)}=0
$$

where the expression $\mathcal{C}=0$ represents the condition and all of its differential consequences up to the order of the equation, see $[12,18]$ and the references therein for more results. Equation (4) gives nonlinear determining equations for $\boldsymbol{\xi}$ and $\phi$ which 


\section{The discretized Boussinesq equation and its conditional symmetry reduction}

provide at the same time the classical and non-classical symmetries. As $\mathcal{C}=0$ appears in (4) as a condition imposed on the determining equations one has called the resulting symmetries conditional symmetries [6,18, 19,33,41].

It has been shown in $[31,32]$ that a $\operatorname{PDE} \mathcal{E}=0$ can be written in terms of the invariants $I_{j}$ of its conditional symmetries $\hat{X}$ as

$$
\left.\mathcal{E}\left(\left\{I_{j}\right\}\right)\right|_{\mathcal{C}=0}=0, \quad j=0,1, \ldots
$$

Let us recall here the conditional symmetries of the Boussinesq equation and how one re-constructs the Boussinesq equations from the invariants of the conditional symmetries.

The Boussinesq equation

$$
u_{y y}+u u_{x x}+u_{x}^{2}+u_{x x x x}=0
$$

was introduced in 1871 by Boussinesq to describe the propagation of long waves in shallow water $[8,9]$ and it is of considerable physical and mathematical interest $[46,48,50,51]$.

The Lie point symmetries are given by [40]

$$
D=x \partial_{x}+2 y \partial_{y}-2 u \partial_{u}, \quad P_{1}=\partial_{x}, \quad P_{2}=\partial_{y} .
$$

The conditional symmetries are divided in two classes. If $\eta \neq 0$ in the vector field

$$
\hat{X}=\xi(x, y, u) \partial_{x}+\eta(x, y, u) \partial_{y}+\phi(x, y, u) \partial_{u}
$$

we can always put $\eta=1$. If $\eta=0$ and $\xi \neq 0$, we can put $\xi=1$. The conditional symmetries of the Boussinesq equation for $\eta \neq 0$ were obtained by Levi and Winternitz in [33], and by Clarkson and Kruskal in [14] by non group techniques. The singular case $\eta=0$, vector fields $\hat{X}_{6}$ and $\hat{X}_{7}$, can be found in a review by Clarkson [13]:

$$
\begin{aligned}
\hat{X}_{1} & =\partial_{y}+y \partial_{x}-2 y \partial_{u}, \\
\hat{X}_{2} & =\partial_{y}-\frac{x}{y} \partial_{x}+\left(\frac{2}{y} u+\frac{6}{y^{3}} x^{2}\right) \partial_{u}, \\
\hat{X}_{3} & =\partial_{y}+\left(-\frac{x}{y}+y^{4}\right) \partial_{x}+\left(\frac{2}{y} u+\frac{6}{y^{3}} x^{2}-2 y^{2} x-4 y^{7}\right) \partial_{u}, \\
\hat{X}_{4} & =\partial_{y}+\left(\frac{x}{2 y}+y\right) \partial_{x}-\frac{1}{y}\left(u+2 x+4 y^{2}\right) \partial_{u}, \\
\hat{X}_{5} & =\partial_{y}+\frac{\dot{\wp}}{2 \wp}(x+\beta W) \partial_{x}-\left[\frac{\dot{\wp}}{\wp} u+3 x^{2} \dot{\wp}+\frac{\beta}{2}\left(\frac{1}{\wp}+12 \dot{\wp} W\right)\right. \\
& \left.+\frac{\beta^{2}}{2} W\left(\frac{1}{\wp}+6 \dot{\wp} W\right)\right] \partial_{u}, \quad W(y)=\int_{0}^{y} \frac{\wp(s)}{\dot{\wp}(s)^{2}} d s, \\
\hat{X}_{6} & =\partial_{x}+\left(\frac{2}{x} u+\frac{48}{x^{3}}\right) \partial_{u}, \\
\hat{X}_{7} & =\partial_{x}+\left(-2 x Q+c_{1} Q+c_{2} Q \int_{0}^{y} \frac{d s}{Q(s)^{2}}\right) \partial_{u},
\end{aligned}
$$

where $Q=\wp\left(y+c_{3} ; 0, g_{3}\right)$ and $\wp$ is a Weierstrass elliptic function. 
The discretized Boussinesq equation and its conditional symmetry reduction

From the way they are constructed it follows that the generators $\hat{X}_{1}, \cdots, \hat{X}_{7}$ are defined in (9)-(15) up to an arbitrary multiplicative function.

In the following we present an overview of the results obtained earlier [31, 32], namely the construction of the Boussinesq equation from the invariants of three chosen conditional symmetries and the reduction with respect to these conditional symmetries. These are the cases we shall discretize in Section 3.

\subsection{Conditional invariant Boussinesq equation associated to $\hat{X}_{1}$}

The invariants of $\hat{X}_{1}=\partial_{y}+y \partial_{x}-2 y \partial_{u}$ which will be of interest here are:

$$
\begin{aligned}
& I_{0}=x-\frac{1}{2} y^{2}, \quad I_{1}=2 x+u, \quad I_{2}=u_{x}, \quad I_{3}=2 y+y u_{x}+u_{y}, \\
& I_{4}=u_{x x}, \quad I_{5}=u_{y y}+2 y u_{x y}+y^{2} u_{x x}, \quad I_{6}=u_{x x x x} .
\end{aligned}
$$

and the condition is given by $I_{3}=0$ i.e. $C=2 y+y u_{x}+u_{y}=0$. Then the Boussinesq equation, written in terms of the invariants is:

$$
\left(I_{0}+I_{1}\right) I_{4}+I_{2}^{2}+I_{5}+\left.I_{6}\right|_{C_{x}=0}=0
$$

As $C_{x}=u_{x y}+y u_{x x}$, we have:

$u_{y y}+2 y\left(u_{x y}+y u_{x x}\right)+u u_{x x}+u_{x x x x}+\left.u_{x}^{2}\right|_{C_{x}=0}=u_{y y}+u u_{x x}+u_{x x x x}+u_{x}^{2}=0$.

Symmetry variables associated to $\hat{X}_{1}$ are:

$$
z=x-\frac{1}{2} y^{2}, \quad u(x, y)=w(z)-y^{2} .
$$

and the equation in these new variables is $\left(w^{\prime}=\mathrm{d} w / \mathrm{d} z\right)$ :

$$
w^{i v}+w w^{\prime \prime}+w^{\prime 2}-w^{\prime}-2=0 .
$$

After a quadrature (20) becomes:

$$
w^{\prime \prime \prime}+w w^{\prime}-w-2 z+A=0,
$$

where $A$ is an integration constant. Equation (21) can be integrated to a second order differential equation and solved in terms of $P_{I I}$ transcendents (see [14]).

\subsection{Conditional invariant Boussinesq equation associated to $\hat{X}_{2}$}

The invariants of interest in the case of $\hat{X}_{2}=\partial_{y}-\frac{x}{y} \partial_{x}+\left(\frac{2}{y} u+\frac{6 x^{2}}{y^{3}}\right) \partial_{u}$ are

$I_{0}=x y, \quad I_{1}=x^{2} u+\frac{x^{4}}{y^{2}}, \quad I_{2}=x^{3} u_{x}+\frac{2 x^{4}}{y^{2}}$,

$I_{3}=x^{2}\left(u_{y}-\frac{x}{y} u_{x}-\frac{2 u}{y}-\frac{6 x^{2}}{y^{3}}\right), \quad I_{4}=x^{4} u_{x x}+\frac{2 x^{4}}{y^{2}}, \quad I_{5}=x^{6} u_{x x x x}$.

The condition is given by $I_{3}=0$, or by any other expression $C=f(x, y) I_{3}=0$. We choose

$$
C=y u_{y}-x u_{x}-2 u-6 x^{2} / y^{2}=0 .
$$


The discretized Boussinesq equation and its conditional symmetry reduction

Then the Boussinesq equation written in terms of the invariants is:

$$
I_{1} I_{4}+I_{5}+I_{2}^{2}+\left.Q\right|_{\{C=0\}}=x^{6}\left(u_{y y}+u u_{x x}+u_{x x x x}+u_{x}^{2}\right)
$$

where by $\{C=0\}$ we mean the substitution of $C=0$ and all its differential consequences. In this case the function of $C$ to be added is $Q=x^{6}\left(x C_{x}+y C_{y}+C\right) / y^{2}$.

The symmetry variables associated to $\hat{X}_{2}$ are

$$
z=x y, \quad u(x, y)=y^{2} w(z)-\frac{x^{2}}{y^{2}} .
$$

The Boussinesq equation in the symmetry variables (25) is:

$$
w^{i v}+w w^{\prime \prime}+w^{2}=0
$$

and after two integration we get:

$$
w^{\prime \prime}+\frac{1}{2} w^{2}=c_{1} z+c_{0}
$$

whose solution is:

(i) If $c_{1}=0$, an elliptic function

(ii) If $c_{1} \neq 0$, a Painlevé I function

\subsection{Conditionally invariant Boussinesq equation associated to $\hat{X}_{6}$}

To construct the Boussinesq equation we need the following invariants of $\hat{X}_{6}=$ $\partial_{x}+\left(\frac{2}{x} u+\frac{48}{x^{3}}\right) \partial_{u}$ :

$I_{0}=y, \quad I_{1}=\frac{1}{x^{4}}\left(12+x^{2} u\right), \quad I_{2}=\frac{1}{x^{5}}\left(x^{3} u_{x}-2 x^{2} u-48\right), \quad I_{3}=\frac{1}{x^{2}} u_{y y}$.

We will use the characteristic of $\hat{X}_{6}$, proportional to $I_{2}$, in the construction of the condition for the Boussinesq equation:

$$
C=x^{2} I_{2}=u_{x}+\frac{2 u}{x}-\frac{48}{x^{3}}=0 .
$$

Then the Boussinesq equation, written in terms of the invariants is:

$$
6 I_{1}^{2}+I_{3}+\left.Q\right|_{\{C=0\}}=\frac{1}{x^{2}}\left(u_{y y}+u u_{x x}+u_{x x x x}+u_{x}^{2}\right),
$$

where the function $Q$, written in terms of the condition $C$ is:

$$
Q=\frac{1}{x^{2}} C_{x x x}+\frac{2}{x^{3}} C_{x x}+\frac{1}{x^{2}}\left(u-\frac{2}{x^{2}}\right) C_{x}+\frac{6}{x^{3}}\left(u+\frac{16}{x^{2}}\right) C+\frac{1}{x^{2}} C^{2} .
$$

The symmetry variables associated to $\hat{X}_{6}$ are:

$$
z=y, \quad u(x, y)=x^{2} w(z)-\frac{12}{x^{2}} .
$$

The Boussinesq equation in the symmetry variables (32) is:

$$
w^{\prime \prime}+6 w^{2}=0
$$

with a solution given by $w=-\wp\left(z+c_{1} ; 0, c_{2}\right)$. 
The discretized Boussinesq equation and its conditional symmetry reduction

\section{The conditionally discretized Boussinesq equations}

The aim of this section is to discretize the Boussinesq equation preserving given conditional symmetries. We include the necessary detailed description and formulas of the discretization formalism used here in the Appendix A, for the convenience of the reader.

Symmetry preserving discretization of the partial differential equation requires that the $\mathrm{P} \Delta \mathrm{E}$ in the continuous limit goes to the original PDE, and that it is invariant under the given symmetry generator prolonged appropriately. Additionally, one must introduce a symmetry invariant lattice, since the fixed nontransformable lattice allows only dilation symmetries of the dependent variable.

Thus we proceed in the following way:

(i) Construct a discrete lattice that is invariant under the given conditional symmetry.

(ii) Discretize the PDE over the obtained lattice, using the same invariants, mutatis mutandis, of the conditional symmetry as introduced in the continuous case (see Section 2).

(iii) Go over to the symmetry variables as in most of the cases the resulting equations and lattices are simpler.

We will present only the cases $\hat{X}_{1}$ and $\hat{X}_{2}$ (where $\eta=1$ ) and the case $\hat{X}_{6}$ (where $\eta=0$ ) to show that conditional discretization can be carried out. The discussion of the other cases follows the same lines.

\section{1. $\hat{X}_{1}$ preserving discretization of the Boussinesq equation}

When applied to dependent and independent variables living on a lattice, the $\hat{X}_{1}$ reads:

$$
\hat{X}_{n m}^{1}=y_{n m} \partial_{x_{n m}}+\partial_{y_{n m}}-2 y_{n m} \partial_{u_{n m}}
$$

thus $\xi_{n m}=y_{n m}, \eta_{n m}=1$ and $\phi_{n m}=-2 y_{n m}$. To define the lattice we construct the prolongation of $\hat{X}_{n m}^{1}$ on points $(n, m),(n+1, m),(n, m+1)$ and $(n+1, m+1)$ as given in (A.14):

$$
\begin{aligned}
& \zeta_{n+i, m+j}^{(x)}=y_{n+i+1, m+j}-y_{n+i, m+j}=\sigma_{n+i, m+j}^{(y)}, \\
& \zeta_{n+i, m+j}^{(y)}=0, \\
& \chi_{n+i, m+j}^{(x)}=y_{n+i, m+j+1}-y_{n+i, m+j}=h_{n+i, m+j}^{(y)}, \\
& \chi_{n+i, m+j}^{(y)}=0, \quad(i, j) \rightarrow(0,1) .
\end{aligned}
$$

The lattice invariants are obtained solving the characteristic equations

$$
\frac{d x_{n m}}{y_{n m}}=\frac{d y_{n m}}{1}=\frac{d h_{n+i, m+j}^{x}}{\sigma_{n+i, m+j}^{y}}=\frac{d \sigma_{n+i, m+j}^{x}}{h_{n+i, m+j}^{y}} .
$$

We get that $h_{n m}^{y}$ and $\sigma_{n m}^{y}$ are invariants $\forall m, n$. We simplify the lattice further by choosing

$$
h_{n m}^{y}=k, \quad \sigma_{n m}^{y}=\sigma, \forall n, m,
$$


The discretized Boussinesq equation and its conditional symmetry reduction where $k$ and $\sigma$ are constants. Furthermore, from (36) we get

$$
\begin{aligned}
& x_{n m}-\frac{1}{2} y_{n m}^{2}=\mathcal{J}_{n m}^{0}, \\
& h_{n+i, m+j}^{x}-y_{n m} \sigma=\mathcal{J}_{i j}^{1}, \\
& \sigma_{n+i, m+j}^{x}-y_{n m} k=\mathcal{J}_{i j}^{2},
\end{aligned}
$$

where $\mathcal{J}_{n m}^{0}, \mathcal{J}_{i j}^{1}, \mathcal{J}_{i j}^{2}$ are invariants obtained from (36) $\forall m, n, i, j$. Choosing $\sigma=0$ and $\mathcal{J}_{i j}^{2}=0$ we obtain $h_{n+i, m+j}^{x}=h$ with $h$ constant and $\sigma_{n+i, m+j}^{x}=k y_{n m} \forall i, j$. Thus the invariant lattice is given by:

$$
h_{n+i, m+j}^{y}=k, \sigma_{n+i, m+j}^{y}=0, h_{n+i, m+j}^{x}=h, \sigma_{n+i, m+j}^{x}=k y_{n m}
$$

where $h$ and $k$ are the constant invariant spacings in the two independent directions (see Figures A1 and A2 in the Appendix). In the following we will use the notation (if there is no risk of confusion), $x_{n m}=x, y_{n m}=y$ and $u_{n m}=u$. The lattice is non orthogonal since $\sigma^{x}$ depends on $y$ but with constant spacing and Schwarzian i.e. such that the second order partial differences commute.

On this lattice the two discrete derivatives (A.7) are:

$$
\mathcal{D}_{x}=\frac{1}{h} \Delta_{n}, \quad \mathcal{D}_{y}=\frac{1}{k} \Delta_{m}-y \mathcal{D}_{x}
$$

The relevant discrete invariants are:

$$
\begin{aligned}
& \mathcal{I}^{(0)}=\mathcal{J}^{0}=x-\frac{1}{2} y^{2}, \quad \mathcal{I}^{(1)}=2 x+u, \quad \mathcal{I}^{(2)}=\mathcal{D}_{x} u, \quad \mathcal{I}^{(3)}=2 y+y \mathcal{D}_{x} u+\mathcal{D}_{y} u, \\
& \mathcal{I}^{(4)}=\mathcal{D}_{x}^{2} u, \quad \mathcal{I}^{(5)}=\mathcal{D}_{y}^{2} u+y^{2} \mathcal{D}_{x}^{2} u+2 y \mathcal{D}_{x} \mathcal{D}_{y} u, \quad \mathcal{I}^{(6)}=\mathcal{D}_{x}^{4} u .
\end{aligned}
$$

They can also be obtained by taking into account that $\mathcal{I}^{(i)}=\mathcal{I}_{n m}^{(i)}, \forall n, m$. For example:

$$
\mathcal{I}_{n m}^{(1)}=2 x_{n m}+u_{n m}
$$

Then from the invariants

$$
\mathcal{I}_{n m}^{(1)}=2 x_{n m}+u_{n m}, \quad \mathcal{I}_{n+1, m}^{(1)}=2 x_{n+1, m}+u_{n+1, m}
$$

by substraction and division by the invariant $\mathcal{J}_{i j}^{1}=h$ we easily get using (41) and (42) that $\mathcal{I}^{(2)}$ given by (43) is an invariant. A similar construction will provide all other higher invariants.

The condition, like in the continuous case, is just $\mathcal{C}=\mathcal{I}^{(3)}=0$ and by discrete differentiation we get

$$
\mathcal{D}_{x} \mathcal{C}=\mathcal{D}_{x} \mathcal{D}_{y} u+y \mathcal{D}_{x}^{2} u=0
$$

The conditional symmetry $\hat{X}_{1}$ preserving discretization of the Boussinesq equation is then given by:

$$
\begin{aligned}
& \left(\mathcal{I}^{(1)}+\mathcal{I}^{(0)}\right) \mathcal{I}^{(4)}+\left(\mathcal{I}^{(2)}\right)^{2}+\mathcal{I}^{(5)}+\left.\mathcal{I}^{(6)}\right|_{\mathcal{D}_{x} C=0} \\
& =\left(y^{2}+u\right) \mathcal{D}_{x}^{2} u+\mathcal{D}_{y}^{2} u+y^{2} \mathcal{D}_{x}^{2} u+2 y \mathcal{D}_{x} \mathcal{D}_{y} u+\mathcal{D}_{x}^{4} u+\left.\left(\mathcal{D}_{x} u\right)^{2}\right|_{\mathcal{D}_{x} \mathcal{D}_{y} u+y} \mathcal{D}_{x}^{2} u=0 \\
& =\mathcal{D}_{y}^{2} u+u \mathcal{D}_{x}^{2} u+\mathcal{D}_{x}^{4} u+\left(\mathcal{D}_{x} u\right)^{2}=0
\end{aligned}
$$


The discretized Boussinesq equation and its conditional symmetry reduction

which, as a difference equation, reads

$$
\begin{aligned}
& \frac{1}{k^{2}}\left(u_{n, m+2}-2 u_{n, m+1}+u_{n m}\right)-\frac{2 y}{h k}\left(u_{n+1, m+1}-u_{n+1, m}-u_{n, m+1}+u_{n m}\right) \\
& +\frac{y^{2}}{h^{2}}\left(u_{n+2, m}-2 u_{n+1, m}+u_{n m}\right)+\frac{u_{n m}}{h^{2}}\left(u_{n+2, m}-2 u_{n+1, m}+u_{n m}\right) \\
& +\frac{1}{h^{4}}\left(u_{n+4, m}-4 u_{n+3, m}+6 u_{n+2, m}-4 u_{n+1, m}+u_{n m}\right) \\
& +\frac{1}{h^{2}}\left(u_{n+1, m}-u_{n m}\right)^{2}-\frac{1}{h}\left(u_{n+1, m+1}-u_{n, m+1}\right)=0
\end{aligned}
$$

From the invariants $\mathcal{I}^{(0)}$ and $\mathcal{I}^{(1)}$ of $\hat{X}_{n m}^{1}$ we can introduce a new dependent variable $w_{n m}\left(x_{n m}, y_{n m}\right)=w$ given by

$$
w=u+y^{2} .
$$

From (47) the equation satisfied by $w$ is:

$$
\mathcal{D}_{x}^{4} w+\mathcal{D}_{y}^{2} w+\left(\mathcal{D}_{x} w\right)^{2}+\left(w-y^{2}\right) \mathcal{D}_{x}^{2} w-2=0
$$

or, explicitly,

$$
\begin{aligned}
& \frac{1}{h^{4}}\left(w_{n+4, m}-4 w_{n+3, m}+6 w_{n+2, m}-4 w_{n+1, m}+w_{n m}\right) \\
& \quad+\frac{1}{h^{2}}\left(w_{n+1, m}^{2}+w_{n m}\left(w_{n+2, m}-4 w_{n+1, m}+2 w_{n m}\right)\right) \\
& \quad-\frac{2 y}{h k}\left(w_{n+1, m+1}-w_{n+1, m}-w_{n, m+1}+w\right)-\frac{1}{h}\left(w_{n+1, m+1}-w_{n, m+1}\right) \\
& \quad+\frac{1}{k^{2}}\left(w_{n, m+2}-2 w_{n, m+1}+w_{n m}\right)-2=0 .
\end{aligned}
$$

By going from the independent variable $x$ to the new independent invariant variable $z_{n m}=z$ given by

$$
z=x-\frac{1}{2} y^{2},
$$

we get that the following relations turn out to be true:

$$
\begin{aligned}
& z_{n+1, m}-z_{n m}=h_{n m}^{z}=x_{n+1, m}-x_{n m}-\frac{1}{2}\left(y_{n+1, m}^{2}-y_{n m}^{2}\right)=h, \\
& z_{n, m+1}-z_{n m}=\sigma_{n m}^{z}=x_{n, m+1}-x_{n m}-\frac{1}{2}\left(y_{n, m+1}^{2}-y_{n m}^{2}\right)=-\frac{1}{2} k^{2} .
\end{aligned}
$$

The fact that in the symmetry variables the lattice spacing is constant, even if non orthogonal, is not an apriori obvious result. However it derives from the fact that in the symmetry variables the infinitesimal generator reduces to $\hat{X}_{1}=\partial_{y_{n m}}$.

Then the differences of the function $w(x, y)=v(z, t)$ (where $t_{n m}=y_{n m}$ and we changed the name of the dependent variable from $w$ to $v$ to stress the change of variables), are:

$$
\begin{aligned}
& \mathcal{D}_{z} v=\frac{v_{n+1, m}-v_{n m}}{h}, \\
& \mathcal{D}_{t} v=k \frac{v_{n+1, m}-v_{n m}}{2 h}+\frac{v_{n, m+1}-v_{n m}}{k}
\end{aligned}
$$


The discretized Boussinesq equation and its conditional symmetry reduction

so that

$$
\begin{aligned}
& v_{n+1, m}=v_{n m}+h \mathcal{D}_{z} v, \\
& v_{n, m+1}=v_{n m}+k \mathcal{D}_{t} v-\frac{k^{2}}{2} \mathcal{D}_{z} v .
\end{aligned}
$$

Then we can rewrite the differences with respect to $x, y$ in terms of $z, t$ as:

$\mathcal{D}_{x} w=\frac{1}{h}\left(v_{n+1, m}-v_{n m}\right)=\mathcal{D}_{z} v$

$\mathcal{D}_{y} w=\frac{1}{k}\left(v_{n, m+1}-v_{n m}\right)-\frac{y}{h}\left(v_{n+1, m}-v_{n m}\right)=\mathcal{D}_{t} v-\left(y+\frac{k}{2}\right) \mathcal{D}_{z} v$.

The coefficients of the discrete derivatives provide the Jacobian of the transformation $(49,52)$. Any other higher order difference can be equally computed. Those we will need for our work are:

$\mathcal{D}_{x}^{2} v=\mathcal{D}_{z}^{2} v, \quad \mathcal{D}_{x}^{4} v=\mathcal{D}_{z}^{4} v$,

$\mathcal{D}_{y}^{2} v=\left(y^{2}+k y+\frac{3 k^{2}}{4}\right) \mathcal{D}_{z}^{2} v-2(y+k) \mathcal{D}_{z} \mathcal{D}_{t} v+\mathcal{D}_{t}^{2} v-\mathcal{D}_{z} v$

Substituting (57) in (50) we get the Boussinesq equation in the invariant discrete variable

$\mathcal{D}_{z}^{4} v+\left(v+k t+\frac{3 k^{2}}{4}\right) \mathcal{D}_{z}^{2} v-2(t+k) \mathcal{D}_{z} \mathcal{D}_{t} v+\mathcal{D}_{t}^{2} v+\mathcal{D}_{z} v+\left(\mathcal{D}_{z} v\right)^{2}-2=0$

where $v=v_{n m}, t=t_{n m}, z=z_{n m}$.

This will be the starting point for the symmetry reduction which will be carried out in the following Section.

\section{2. $\hat{X}_{2}$ preserving discretization of the Boussinesq equation}

In this case we have

$$
\hat{X}_{n m}^{2}=\partial_{y_{n m}}-\frac{x}{y} \partial_{x_{n m}}+\left(\frac{2}{y_{n m}} u_{n m}+\frac{6}{y_{n m}^{3}} x_{n m}^{2}\right) \partial_{u_{n m}} .
$$

Repeating the detailed calculation we made in the previous subsection we find the invariant lattice given by:

$h_{n m}^{y}=k, \quad \sigma_{n m}^{y}=0, \quad h_{n+i, m+j}^{x}=\frac{h}{y_{n m}}, \quad \sigma_{n+i, m+j}^{x}=-\frac{k x_{n m}}{y_{n, m+1}} \quad \forall i, j, m, n$

where $h$ and $k$ are two constants related to the invariant spacing in the two independent directions (see Figures A1 and A2 in the Appendix) and we will set as before, when possible, $x_{n m}=x, y_{n m}=y$ and $u_{n m}=u$. The lattice is non orthogonal, with non constant spacing in the $x$ direction and non Schwarzian.

On this lattice the two discrete derivatives (A.7) are:

$$
\mathcal{D}_{x}=\frac{y}{h} \Delta_{n}, \quad \mathcal{D}_{y}=\frac{1}{k} \Delta_{m}+\frac{x}{y+k} \mathcal{D}_{x} .
$$


The discretized Boussinesq equation and its conditional symmetry reduction

The invariants we need for our construction are:

$$
\begin{aligned}
\mathcal{I}^{(0)} & =x y, \quad \mathcal{I}^{(1)}=x^{2} u+\frac{x^{4}}{y^{2}}, \quad \mathcal{I}^{(2)}=x^{3} \mathcal{D}_{x} u+\frac{2 x^{4}}{y^{2}}+\frac{x^{3} h}{y^{3}} \\
\mathcal{I}^{(3)} & =\frac{x^{2} y^{2}}{(y+k)^{2}} \mathcal{D}_{y} u-\frac{x^{3} y^{2}}{(y+k)^{3}} \mathcal{D}_{x} u-\frac{x^{2}(k+2 y)}{(y+k)^{2}} u \\
& -\frac{x^{4}(k+2 y)\left(k^{2}+k y+y^{2}\right)\left(k^{2}+3 k y+3 y^{2}\right)}{y^{2}(y+k)^{6}} \\
\mathcal{I}^{(4)} & =x^{4} \mathcal{D}_{x}^{2} u+\frac{2 x^{4}}{y^{2}}, \quad \mathcal{I}^{(5)}=x^{6} \mathcal{D}_{x}^{4} u
\end{aligned}
$$

It is easy to check that in the continuous limit $(h, k) \rightarrow(0,0))$ :

$$
\mathcal{I}^{(i)} \rightarrow I_{i} \quad i=1, \ldots, 5
$$

where $I_{i}$ are the invariants of the continuous case $(22)$. Then the expression $\mathcal{I}^{(1)} \mathcal{I}^{(4)}+$ $\mathcal{I}^{(5)}+\left(\mathcal{I}^{(2)}\right)^{2}$ has the appropriate continuous limit too when $(h, k) \rightarrow(0,0)$ :

$$
\mathcal{I}^{(1)} \mathcal{I}^{(4)}+\mathcal{I}^{(5)}+\left(\mathcal{I}^{(2)}\right)^{2} \rightarrow I_{1} I_{4}+I_{5}+I_{2}^{2}
$$

Regarding the condition, we choose, (for the sake of simplicity)

$$
\begin{aligned}
\mathcal{C}= & \frac{(y+k)^{2}}{x^{2} y} \mathcal{I}^{(3)} \\
& =y \mathcal{D}_{y} u-\frac{x y \mathcal{D}_{x} u}{y+k}-\frac{2 y+k}{y} u-\frac{x^{2}(k+2 y)\left(k^{2}+k y+y^{2}\right)\left(k^{2}+3 k y+3 y^{2}\right)}{y^{3}(y+k)^{4}} .
\end{aligned}
$$

with $\mathcal{C} \rightarrow C(23)$ when $(h, k) \rightarrow(0,0)$. We define:

$$
\mathcal{Q}=\frac{x^{6}}{y^{2}}\left(y \mathcal{D}_{y} \mathcal{C}+x \mathcal{D}_{x} \mathcal{C}+\mathcal{C}\right)
$$

The explicit expression of $\mathcal{I}^{(1)} \mathcal{I}^{(4)}+\mathcal{I}^{(5)}+\left(\mathcal{I}^{(2)}\right)^{2}+\mathcal{Q}$, after dividing by $x^{6}$, is:

$$
\begin{aligned}
\mathcal{D}_{x}^{4} u & +\left(\mathcal{D}_{x} u\right)^{2}+u \mathcal{D}_{x}^{2} u+\mathcal{D}_{y}^{2} u \\
& +\frac{k}{y} \mathcal{D}_{y}^{2} u-\frac{k^{2} x}{y(y+k)(y+2 k)} \mathcal{D}_{x y} u \\
& +\frac{2 h(y+k)^{2}(y+2 k)+k^{2} x\left(y^{2}-3 k y-2 k^{2}\right)}{y^{3}(y+k)^{2}(y+2 k)} \mathcal{D}_{x} u-\frac{k}{y(y+k)} \mathcal{D}_{y} u \\
& +\frac{k x^{2}\left(y^{2}+4 k y+2 k^{2}\right)-h x\left(y^{2}+3 k y+k^{2}\right)}{y^{2}(y+k)^{2}(y+2 k)} \mathcal{D}_{x}^{2} u-\frac{k^{2}}{y^{3}(y+k)} u \\
& +\frac{1}{y^{6}(y+k)^{5}(y+2 k)^{4}}\left(k^{10}\left(-16 h x-48 x^{2} y\right)+k^{9}\left(16 h^{2}-96 h x y-352 x^{2} y^{2}\right)\right. \\
& +k^{8}\left(112 h^{2} y-264 h x y^{2}-1192 x^{2} y^{3}\right)+k^{7}\left(344 h^{2} y^{2}-488 h x y^{3}-2488 x^{2} y^{4}\right) \\
& +k^{6}\left(608 h^{2} y^{3}-761 h x y^{4}-3571 x^{2} y^{5}\right)+k^{5}\left(681 h^{2} y^{4}-1036 h x y^{5}-3664 x^{2} y^{6}\right) \\
& +k^{4}\left(501 h^{2} y^{5}-1087 h x y^{6}-2677 x^{2} y^{7}\right)+k^{3}\left(242 h^{2} y^{6}-770 h x y^{7}-1312 x^{2} y^{8}\right) \\
& +k^{2}\left(74 h^{2} y^{7}-335 h x y^{8}-379 x^{2} y^{9}\right)+k\left(13 h^{2} y^{8}-80 h x y^{9}-48 x^{2} y^{10}\right) \\
& \left.+h^{2} y^{9}-8 h x y^{10}\right)=0,
\end{aligned}
$$


with lattice parameters (60).

Let us introduce the symmetry dependent variable of $\hat{X}_{n m}^{2} w_{n m}(x, y) \equiv w$ given by

$$
w=\frac{u}{y^{2}}+\frac{x^{2}}{y^{4}}
$$

Although (67) written in terms of $w$ is very complicated the equation greatly simplifies when we go to the independent symmetry variables $z_{n m}=z, t_{n m}=t$

$$
z=x y, \quad t=y .
$$

In this case we have the following relations:

$$
\begin{aligned}
& z_{n+1, m}-z=h_{n m}^{z}=x_{n+1, m} y_{n+1, m}-x y=h, \\
& z_{n, m+1}-z=\sigma_{n m}^{z}=x_{n, m+1} y_{n, m+1}-x y=0, \\
& t_{n+1, m}-t=\sigma_{n m}^{t}=y_{n+1, m}-y=0 \\
& t_{n, m+1}-t=h_{n m}^{t}=y_{n, m+1}-y=k
\end{aligned}
$$

which imply, as it should be, that in the new variables the lattice is orthogonal and uniform in both directions. The variations of the dependent function $w(x, y)=$ $v_{n m}(z, t)=v$ are:

$$
\begin{aligned}
& \mathcal{D}_{z} v=\frac{v_{n+1, m}-v_{n m}}{h}, \\
& \mathcal{D}_{t} v=\frac{v_{n, m+1}-v_{n m}}{k}
\end{aligned}
$$

and then we obtain the following expressions for the shifted dependent variable:

$$
\begin{aligned}
& v_{n+1, m}=v_{n m}+h \mathcal{D}_{z} v, \\
& v_{n, m+1}=v_{n m}+k \mathcal{D}_{t} v .
\end{aligned}
$$

Then the differences with respect to $x, y$ becomes:

$\mathcal{D}_{x} w=y \frac{w_{n+1, m}-w_{n m}}{h}=y \mathcal{D}_{z} v=t \mathcal{D}_{z} v$

$\mathcal{D}_{y} w=\frac{w_{n, m+1}-w_{n m}}{k}+\frac{x y}{y+k} \frac{w_{n+1, m}-w_{n m}}{h}=\frac{x y}{y+k} \mathcal{D}_{z} v+\mathcal{D}_{t} v=\frac{t z}{t+k} \mathcal{D}_{z} v+\mathcal{D}_{t} v$

Any other higher order difference can be equally computed. Then (67) reduces to the much simpler expression

$$
\begin{gathered}
t^{6} \mathcal{D}_{z}^{4} v+t^{6}\left(\mathcal{D}_{z} v\right)^{2}+t^{6} v \mathcal{D}_{z}^{2} v+\frac{(t+k)(t+2 k)^{2}}{t} \mathcal{D}_{t}^{2} v+\frac{\left(4 t^{2}+9 k t+5 k^{2}\right)}{t} \mathcal{D}_{t} v \\
+\frac{z\left(2 t^{2}+3 k t+k^{2}\right)}{t} \mathcal{D}_{z t} v=0
\end{gathered}
$$

3.3. $\hat{X}_{6}$ preserving discretization of the Boussinesq equation

In this case,

$$
\hat{X}_{n m}^{6}=\partial_{x_{n m}}+\left(\frac{2}{x_{n m}} u_{n m}+\frac{48}{x_{n m}^{3}}\right) \partial_{u_{n m}}
$$


The discretized Boussinesq equation and its conditional symmetry reduction

and repeating the calculations outlined earlier, the invariant lattice is given by:

$$
h_{n m}^{y}=k, \sigma_{n m}^{y}=0, h_{n m}^{x}=h, \sigma_{n m}^{x}=0,
$$

where $h$ and $k$ are two constant invariant spacing in the two independent directions and, as before, we set, whenever possible, $x_{n m}=x, y_{n m}=y$ and $u_{n m}=u$. The lattice is orthogonal with constant spacing and Schwarzian.

On this lattice the two discrete derivatives (A.7) are:

$$
\mathcal{D}_{x}=\frac{1}{h} \Delta_{n}, \quad \mathcal{D}_{y}=\frac{1}{k} \Delta_{m} .
$$

The relevant discrete invariants are:

$$
\begin{aligned}
& \mathcal{I}^{(0)}=y, \quad \mathcal{I}^{(1)}=\frac{u}{x^{2}}+\frac{12}{x^{4}}, \\
& \mathcal{I}^{(2)}=\frac{1}{(x+h)^{2}} \mathcal{D}_{x} u-\frac{2 x+h}{x^{2}(x+h)^{2}} u-\frac{12(h+2 x)\left(h^{2}+2 x h+2 x^{2}\right)}{x^{4}(x+h)^{4}}, \quad \mathcal{I}^{(3)}=\frac{1}{x^{2}} \mathcal{D}_{y}^{2} u .
\end{aligned}
$$

The discretized Boussinesq equation is obtained considering the invariants:

$$
6\left(\mathcal{I}^{(1)}\right)^{2}+\mathcal{I}^{(3)}=\frac{6}{x^{4}} u^{2}+\frac{144}{x^{6}} u+\frac{864}{x^{8}}+\frac{1}{x^{2}} \mathcal{D}_{y}^{2} u,
$$

when the condition is taken from $\mathcal{I}^{(2)}$ to be

$\mathcal{C}=(x+h)^{2} \mathcal{I}^{(2)}=\mathcal{D}_{x} u-\frac{2 x+h}{x^{2}} u-\frac{12(h+2 x)\left(h^{2}+2 x h+2 x^{2}\right)}{x^{4}(x+h)^{2}}$.

We get the discretized Boussinesq equation as in the continuous case, by adding to (79) the following combination of the condition $\mathcal{C}$ and its discrete derivatives.

$$
\mathcal{Q}=\frac{1}{x^{2}} \mathcal{D}_{x}^{3} \mathcal{C}+\frac{2}{x^{3}} \mathcal{D}_{x}^{2} \mathcal{C}+\frac{1}{x^{2}}\left(u-\frac{2}{x^{2}}\right) \mathcal{D}_{x} \mathcal{C}+\frac{6}{x^{3}}\left(u+\frac{16}{x^{2}}\right) \mathcal{C}+\frac{1}{x^{2}} \mathcal{C}^{2}
$$

The explicit equation is:

$$
\begin{aligned}
\mathcal{D}_{x}^{4} u & +\left(\mathcal{D}_{x} u\right)^{2}+u \mathcal{D}_{x}^{2} u+\mathcal{D}_{y}^{2} u \\
& +\left(\frac{4 h\left(-216 h^{6}-360 h^{5} x+264 h^{4} x^{2}+849 h^{3} x^{3}+633 h^{2} x^{4}+194 h x^{5}+21 x^{6}\right)}{x^{4}(x+h)^{2}(x+2 h)^{2}(x+3 h)^{2}}\right. \\
& \left.-\frac{h\left(2 h^{2}+2 h x+x^{2}\right)}{x^{2}(x+h)^{2}} u\right) \mathcal{D}_{x} u-\frac{h\left(72 h^{3}+210 h^{2} x+119 h x^{2}+18 x^{3}\right)}{x^{2}(x+2 h)^{2}(x+3 h)^{2}} \mathcal{D}_{x}^{2} u \\
& +\frac{h(5 x+18 h)}{x(x+3 h)^{2}} \mathcal{D}_{x}^{3} u+\frac{h\left(h^{3}-2 x^{2}(x+2 h)\right)}{x^{4}(x+h)^{2}} u^{2} \\
& +\frac{2 h u}{x^{6}(x+h)^{4}(x+2 h)^{2}(x+3 h)^{2}}\left(432 h^{9}+1152 h^{8} x-2544 h^{7} x^{2}-16236 h^{6} x^{3}\right. \\
& \left.-33421 h^{5} x^{4}-37700 h^{4} x^{5}-25384 h^{3} x^{6}-10062 h^{2} x^{7}-2139 h x^{8}-186 x^{9}\right) \\
& +\frac{24 h}{x^{8}(x+h)^{4}(x+2 h)^{4}(x+3 h)^{4}(x+4 h)^{2}}\left(124416 h^{15}+476928 h^{14} x-946080 h^{13} x^{2}\right. \\
& +11056320 h^{12} x^{3}-38053920 h^{11} x^{4}-78993456 h^{10} x^{5}-113245354 h^{9} x^{6} \\
& -117950624 h^{8} x^{7}-91213065 h^{7} x^{8}-52724836 h^{6} x^{9}-22680916 h^{5} x^{10} \\
& \left.-7147120 h^{4} x^{11}-1599493 h^{3} x^{12}-240292 h^{2} x^{13}-21692 h x^{14}-888 x^{15}\right)=0 .
\end{aligned}
$$


The discretized Boussinesq equation and its conditional symmetry reduction

We introduce the invariant dependent variable

$$
w\left(x_{n m}, y_{n m}\right) \equiv w=\frac{u}{x^{2}}+\frac{12}{x^{4}},
$$

and independent variables $z_{n m}=y_{n m}=z, t_{n m}=x_{n m}=t$. Rewriting the equation (82) in terms of $w(x, y)=v_{n m}(z, t)$ and their differences we obtain the much simpler equation:

$$
\begin{aligned}
& (t+4 h)^{2} \mathcal{D}_{t}^{4} v+\left(\frac{18 h^{2}}{t}+8 t+33 h\right) \mathcal{D}_{t}^{3} v+\left(t^{2}(t+2 h)^{2} v-\frac{4 h(9 t+14 h)}{t^{2}}\right) \mathcal{D}_{t}^{2} v \\
& +(t+h)^{4}\left(\mathcal{D}_{t} v\right)^{2}+\left(t\left(15 h t+8 t^{2}+6 h^{2}\right) v+\frac{6 h(t+4 h)}{t^{3}}\right) \mathcal{D}_{t} v+t^{2} \mathcal{D}_{z}^{2} v+6 t^{2} v^{2}=0 .
\end{aligned}
$$

\section{Symmetry reduction of the conditional discretization of the Boussinessq equation}

To be able to carry out the symmetry reduction we start from the conditionally discretized Boussinesq equations $(47,74,84)$ rewritten in terms of the corresponding symmetry variables $(z, t, v)$.

\subsection{Case $\hat{X}_{1}$.}

Starting from (58) we can easily see that we can make the restriction

$$
v_{n m}=v_{n}=v \text {. }
$$

Then,

$$
\begin{aligned}
& \mathcal{D}_{z} v=\frac{v_{n+1}-v_{n}}{h}, \\
& \mathcal{D}_{t} v=k \frac{v_{n+1}-v_{n}}{2 h}=\frac{k}{2} \mathcal{D}_{z} v .
\end{aligned}
$$

The other differences are:

$$
\mathcal{D}_{t}^{2} v=\frac{k^{2}}{4} \mathcal{D}_{z}^{2} v, \quad \mathcal{D}_{z t} v=\frac{k}{2} \mathcal{D}_{z}^{2} v
$$

Equation (58) becomes:

$\mathcal{D}_{z}^{4} v+\left(v+k t+\frac{3 k^{2}}{4}\right) \mathcal{D}_{z}^{2} v-2 \frac{k}{2}(t+k) \mathcal{D}_{z}^{2} v+\frac{k^{2}}{4} \mathcal{D}_{z}^{2} v-\mathcal{D}_{z} v+\left(\mathcal{D}_{z} v\right)^{2}-2=0$

which, when $k$ is set equal to zero, becomes the difference equation

$$
\mathcal{D}_{z}^{4} v+v \mathcal{D}_{z}^{2} v-\mathcal{D}_{z} v+\left(\mathcal{D}_{z} v\right)^{2}-2=0
$$

Equation (89) is the reduction of the discretized Boussinesq equation corresponding to the conditional symmetry $\hat{X}_{n m}^{1}$. 


\subsection{Case $\hat{X}_{2}$.}

In $(74)$ we can make the same ansatz as in the $\hat{X}_{1}$ case, $v_{n m}=v_{n}$. Then,

$$
\mathcal{D}_{t} v=0, \quad \mathcal{D}_{t}^{2} v=0, \quad \mathcal{D}_{z t} v=0,
$$

where $z_{n m}=z_{n}=z, t_{n m}=t_{m}=t$. Equation (74) becomes the difference equation:

$$
\left(\mathcal{D}_{z} v\right)^{2}+v \mathcal{D}_{z}^{2} v+\mathcal{D}_{z}^{4} v=0 \text {. }
$$

In the continuous limit we were able to integrate it twice. Using results contained in $[38,39]$ on the variational derivative of a discrete expression like (91) we can show that (91) can not be integrated. This equation, goes in the continuous limit to $P_{I}$ or to a differential equation whose solution is given by an elliptic function. A discrete $P_{I}$ equation [28] is given by

$$
v_{n}\left(v_{n+1}+v_{n}+v_{n-1}-6\right)+n \alpha+3=0,
$$

and it is easy to see that (92) is not a reduction of (91).

\subsection{Case $\hat{X}_{6}$.}

Assuming in (84), $v_{n m}=v_{m}=v$, we get

$$
\mathcal{D}_{t} v=0, \quad \mathcal{D}_{t}^{2} v=0, \quad \mathcal{D}_{t}^{3} v=0, \quad \mathcal{D}_{t}^{4} v=0 .
$$

Equation (84) becomes the discrete difference equation:

$$
\mathcal{D}_{z}^{2} v+6 v^{2}=0
$$

which has the correct continuous limit and whose solution will go in the continuous limit to an elliptic function.

\subsection{Numerical computation}

In the $\hat{X}_{1}$ case we have not at our disposal an exact solution to compare with a numerical approach. So we will discuss the numerical aspects for cases $\hat{X}_{2}$ and $\hat{X}_{6}$ where the exact solutions correspond to $\wp$-Weierstrass functions. The resulting solution can be transformed into solutions of the Boussinesq by writing $u(x, y)$ in terms of $v(z)$ according to $(25)$ and $(32)$.

4.4.1. $\hat{X}_{2}$ conditional symmetry We will consider here the equation we got as reduction of the Boussinesq equation under the conditional symmetry $\hat{X}_{2}(27$.) In the simple case of the Weierstrass equation with $g_{2}=0, g_{3}=2$ it is:

$$
v=-12 \wp(z ; 0,2) \text {. }
$$

We will consider the interval $(1,2.5)$ where the solution is regular (the closest singularities are in $z=0$ and $z \approx 2.72$ ). 
The discretized Boussinesq equation and its conditional symmetry reduction

We can use the discrete scheme we found in Section 4.2, (91), with a Cartesian lattice:

$$
z_{n+1}=z_{n}+h, \quad \mathcal{D}_{z}^{4} v+v \mathcal{D}_{z}^{2} v+\left(\mathcal{D}_{z} v\right)^{2}=0
$$

or, in an explicit form:

$v_{n}=4 v_{n-1}-6 v_{n-2}+4 v_{n-3}-v_{n-4}-h^{2}\left(v_{n-3}^{2}+2 v_{n-4}^{2}-4 v_{n-4} v_{n-3}+v_{n-4} v_{n-2}\right)$.

Taking $h=0.0001$, with the initial conditions given by the exact solution (that is, value of the solution at $z=1, v_{n-3}=1.0001, v_{n-2}=1.0002, v_{n-1}=1.0003$ ) we get the graphics plotted in Figure 1 ( dots) superimposed on the solid curve representing the exact solution.

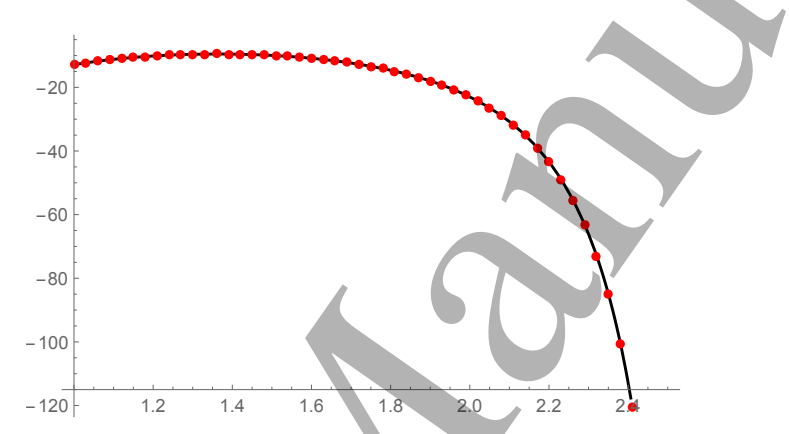

Figure 1. The numeric solution of the discrete reduced Boussinesq equation with the conditional symmetry $X_{2}$ (dots over the exact solution, solid curve).

The $\chi$ estimator, defined as:

$$
\chi=\sqrt{\frac{\sum_{i=1}^{N}\left(V_{i}-v_{i}\right)^{2}}{\sum_{i=1}^{N} V_{i}^{2}}}
$$

where $V_{i}$ stands for the exact value at a point $z_{i}$ and $v_{i}$ for the approximated value at the same point, is:

$$
\chi=3.62737 \times 10^{-3}
$$

in the given interval $(1,2.5)$.

4.4.2. $\hat{X}_{6}$ conditional symmetry Using the conditional symmetry $\hat{X}_{6}$, the Boussinesq equation is directly reduced to the Weierstrass differential equation for the function $v(z)$ (33), as in the previous example. We will consider the solution

$$
v(z)=-\wp(z ; 0,2)
$$

in the interval $(0.50,2.25)$ (again with singularities at $z=0$ and $z \approx 2.72$ ). 

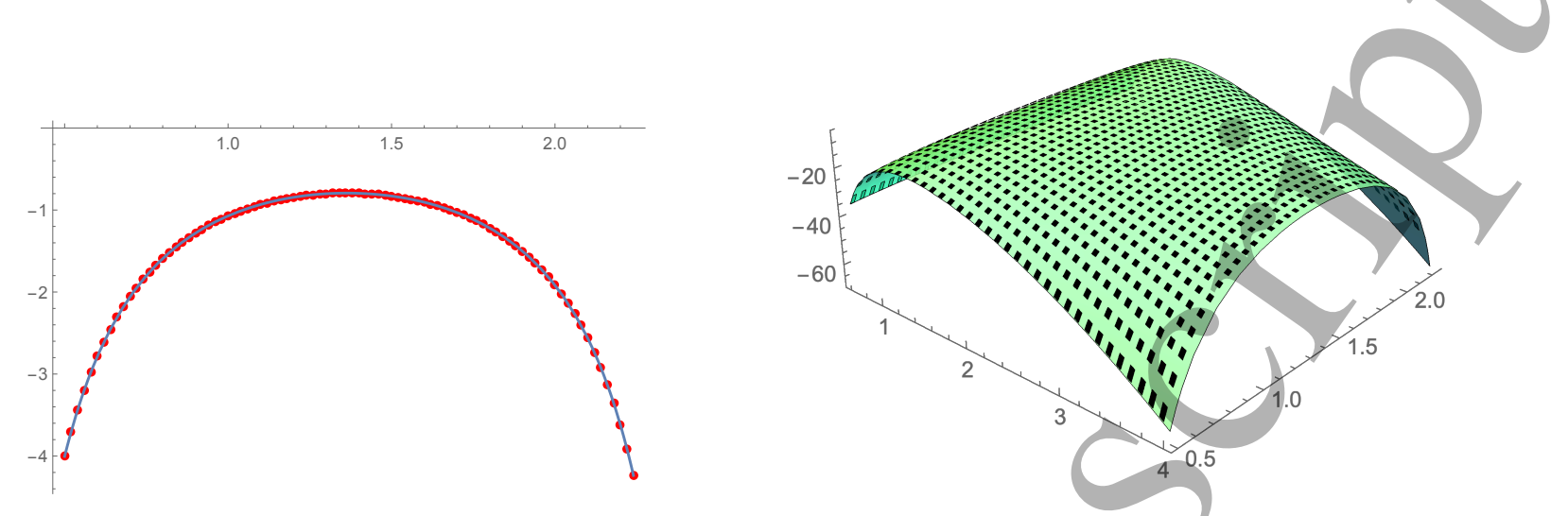

Figure 2. Solutions of the Boussinesq equation corresponding to the reduction using $\hat{X}_{6}$. The solid curve and surface are exact solutions of the reduced equation (33) and the Boussinesq equation (6), respectively. The dots superimposed to the curve and to the surface are computed using our invariant scheme.

Taking $k=10^{-5}$ and initial data from the exact solution above, we represent the approximate result with the exact solution in Figure 2.

The value of the error estimator $\chi$ is in this case, in the interval $(0.50,2.25)$

$$
\chi=1.22 \times 10^{-12} .
$$

As an example we plot in this case in Figure 2 also the solution of the Boussinesq equation as obtained both from the continuous and the discretized reduction.

\section{Conclusions}

In this article we showed that we can carry out conditional symmetry preserving discretization. We consider three conditional symmetries of the Boussinesq equation which give rise to three different lattices, an orthogonal lattice, a lattice depending non trivially on the independent variable and non orthogonal but Schwarzian and a non orthogonal non Schwarzian lattice.

The discrete equations one obtain $(47,67,82)$ are almost always very complicated with many terms depending on the lattice spacing $h$ and $k$ of the two lattice directions which in the continuous limit go to zero providing the continuous Boussinesq equation. In all three cases we are able to rewrite the discrete equation in terms of the symmetry variables of the conditional symmetries. The obtained discrete equations $(58,74,84)$ are greatly simplified, and the three lattices on the plane have constant angles and spacing.

The symmetry reduction can then be carried out and we get three equations which are a simple discretization of the continuous case which however cannot be integrated to second order difference equations as was the case in the corresponding continuous reductions. On these reductions we carried out some numerical calculations to verify the behaviour with respect to the exact solution, when existing. As the lattice spacing 
is always constant, away from the singularity the discrete scheme provides a good approximation of the continuous solution.

An important point on which we are presently working is a more detailed numerical study of the discretized equation we obtained $(47,67,82)$. A second point is the construction of a different discrete derivative as the approximation of the continuous derivative used here is not symmetric with respect to the point $(n, m)$, property which seems to be essential if we expect the discrete equations to be integrable [37], as should be the case of any discretization of the Boussinesq equation. Work on all these problems is in progress.

\section{Acknowledgments}

DL has been supported by INFN IS-CSN4 Mathematical Methods of Nonlinear Physics. DL thanks the Departamento de Física Teórica of Universidad Complutense de Madrid (Spain) for its hospitality. MAR was supported by the Spanish MINECO under project FIS2015-63966-P and thanks the INFN Sezione Roma Tre (Italy) for its hospitality. DL and ZT also thanks the hospitality of CRM, Montreal (Canada).

\section{Appendix A. Description of two dimentional lattices and $\mathrm{P} \Delta \mathrm{Es}$}

Symmetry preserving discretization is the construction of discrete equations which in the continuous limit go over to the given PDE and preserve its geometric structure. As discrete equations defined on a fixed non transformable lattice have only dilation symmetries as their Lie point symmetries, a discrete equation with more general Lie symmetries will have a transformable lattice, will thus be described by what is called a discrete scheme.

In the following we will provide the basis for the description of a difference scheme with a given symmetry. For the sake of completeness we present here results which will be necessary for the calculations performed in Section 3, contained in ref. [29,30].

\section{Appendix A.1. Geometry of 2-dimensional quadrilateral lattices}

A sequence of points $P(x, y)$ in $\mathbf{R}^{2}$ will be characterized by two indices laying on a 2-dimensional quadrilateral lattice.

Four points $\left(x_{n m}, y_{n m}\right),\left(x_{n+1, m}, y_{n+1, m}\right),\left(x_{n, m+1}, y_{n, m+1}\right)$ and $\left(x_{n+1, m+1}, y_{n+1, m+1}\right)$, will define a quadrilateral in $\mathbf{R}^{2}$ (see figure A1). We can define alternative variables, geometrically more relevant, given by the differences,

$$
\begin{aligned}
& x_{n+1, m}-x_{n m}=h_{n m}^{x}, \quad x_{n, m+1}-x_{n m}=\sigma_{n m}^{x}, \\
& y_{n+1, m}-y_{n m}=\sigma_{n m}^{y}, \quad y_{n, m+1}-y_{n m}=h_{n m}^{y},
\end{aligned}
$$

where $h_{n m}^{x}, h_{n m}^{y}, \sigma_{n m}^{x}$ and $\sigma_{n m}^{y}$ are defined graphically in Fig. A2. 
The discretized Boussinesq equation and its conditional symmetry reduction
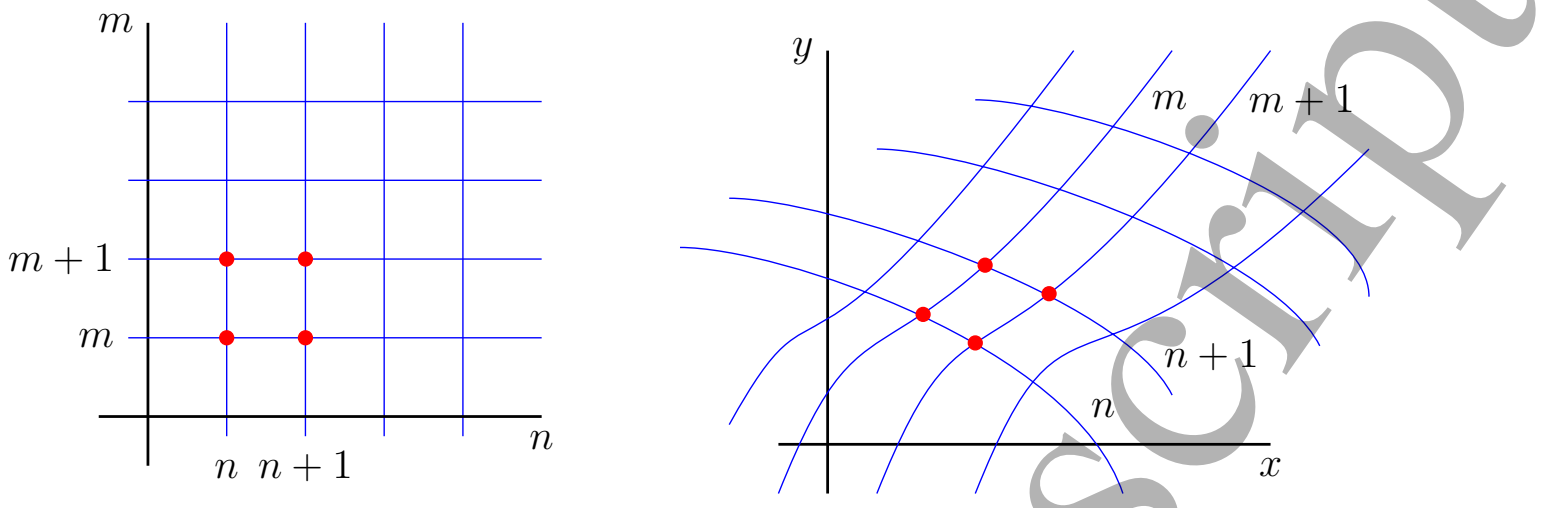

Figure A1. A 2-dimensional lattice of points, on the left the computational and on the right the physical coordinates.

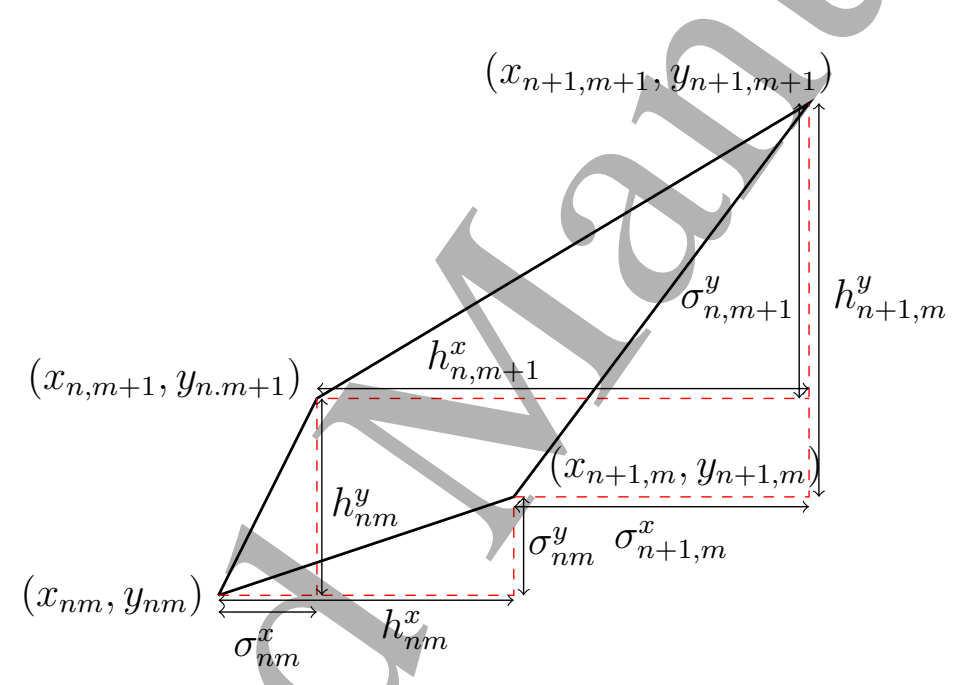

Figure A2. An elementary cell.

As the point $\left(x_{n+1, m+1}, y_{n+1, m+1}\right)$ can be reached following two routes we have the consistency conditions,

$$
h_{n, m+1}^{x}-h_{n m}^{x}=\sigma_{n+1, m}^{x}-\sigma_{n m}^{x}, \quad h_{n+1, m}^{y}-h_{n m}^{y}=\sigma_{n, m+1}^{y}-\sigma_{n m}^{y} .
$$

The vectors defining two adjoint sides of the quadrilateral starting at the point of indices $(n, m)$ must not be parallel,

$$
h_{n m}^{x} h_{n m}^{y}-\sigma_{n m}^{x} \sigma_{n m}^{y} \neq 0
$$

Moreover the expression (A.3) must preserve the sign to avoid the folding of the lattice itself.

The quadrilateral will be a parallelogram if the sides are parallel:

$$
\frac{h_{n, m+1}^{x}}{\sigma_{n, m+1}^{y}}=\frac{h_{n m}^{x}}{\sigma_{n m}^{y}}=a_{n}, \quad \frac{h_{n+1, m}^{y}}{\sigma_{n+1, m}^{x}}=\frac{h_{n m}^{y}}{\sigma_{n m}^{x}}=b_{m} .
$$


The discretized Boussinesq equation and its conditional symmetry reduction

Appendix A.2. Differential and difference operators

In the lowest order terms of the Taylor series expansion of $u_{n+1, m}$ and $u_{n, m+1}$ :

$$
\begin{aligned}
& u_{n+1, m}=u_{n m}+\left(x_{n+1, m}-x_{n m}\right) u_{x}+\left(y_{n+1, m}-y_{n m}\right) u_{y}+O\left(h^{2}\right) \\
& u_{n, m+1}=u_{n m}+\left(x_{n, m+1}-x_{n m}\right) u_{x}+\left(y_{n, m+1}-y_{n m}\right) u_{y}+O\left(h^{2}\right),
\end{aligned}
$$

truncating it at the first order in $h$, we can substitute the first order partial derivatives $u_{x}$ and $u_{y}$ by the approximate discrete first order partial derivatives $\mathcal{D}_{x} u$ and $\mathcal{D}_{y} u$ and (A.5) reads

$$
\begin{aligned}
& u_{n+1, m}=u_{n m}+h_{n m}^{x} \mathcal{D}_{x} u+\sigma_{n m}^{y} \mathcal{D}_{y} u \\
& u_{n, m+1}=u_{n m}+\sigma_{n m}^{x} \mathcal{D}_{x} u+h_{n m}^{y} \mathcal{D}_{y} u .
\end{aligned}
$$

Inverting (A.6) we can approximate the partial differential equations on the lattice by introducing the following discrete partial differential derivatives in the $x$ and $y$ direction

$$
\begin{aligned}
& \mathcal{D}_{x}=\frac{1}{h_{n m}^{x} h_{n m}^{y}-\sigma_{n m}^{x} \sigma_{n m}^{y}}\left(h_{n m}^{y} \Delta_{n}-\sigma_{n m}^{y} \Delta_{m}\right) \\
& \mathcal{D}_{y}=\frac{1}{h_{n m}^{x} h_{n m}^{y}-\sigma_{n m}^{x} \sigma_{n m}^{y}}\left(-\sigma_{n m}^{x} \Delta_{n}+h_{n m}^{x} \Delta_{m}\right)
\end{aligned}
$$

with $T_{n} f_{n m}=f_{n+1, m}, \Delta_{n}=T_{n}-1$, and $T_{m} f_{n m}=f_{n, m+1}, \Delta_{m}=T_{m}-1$. The functions $h_{n m}^{x}, h_{n m}^{y}, \sigma_{n m}^{x}$ and $\sigma_{n m}^{y}$ are such that (A.2) and (A.3) are satisfied.

Proposition 1 Any partial differential equation in two dependent variables can be constructed in terms of the partial difference, operators (A.7) in the directions $x$ and $y$, provided the conditions (A.2, A.3) are satisfied.

\section{Appendix A.3. Schemes for partial difference equations and symmetries}

Here, as an example, we limit ourselves to a scheme in $\mathbf{R}^{2}$ of six points $(n, m),(n+1, m)$, $(n, m+1),(n+2, m),(n, m+2),(n+1, m+1)$, the minimum number of points necessary to get all partial second derivatives as first order approximations. The variables $x, y$ and $u(x, y)$ in all points correspond to 18 data, 12 related to the independent variables and 6 to the dependent one. Having 12 data for the independent variables we can construct from them 10 differences

$x_{00}, y_{00}, h_{0,0}^{x}=x_{10}+x_{00}, h_{00}^{y}=y_{01}-y_{00}, \sigma_{00}^{x}=x_{01}-x_{00}, \sigma_{00}^{y}=y_{10}-y_{00}$,

$h_{10}^{x}=x_{20}-x_{10}, h_{01}^{y}=y_{02}-y_{01}, \sigma_{01}^{x}=x_{02}-x_{01}, \sigma_{10}^{y}=y_{20}-y_{10}$,

$h_{01}^{x}=x_{11}-x_{01}, h_{10}^{y}=y_{11}-y_{10}$,

From the values of the dependent variables in the 6 points we can calculate the 6 quantities

$$
u_{00}, \mathcal{D}_{x} u_{00}, \mathcal{D}_{y} u_{00}, \mathcal{D}_{x}^{2} u_{00}, \mathcal{D}_{y}^{2} u_{00}, \mathcal{D}_{x} \mathcal{D}_{y} u_{00}
$$

$\mathcal{D}_{y} \mathcal{D}_{x} u_{00}$ is not independent from the 6 quantities (A.9). It can be written in terms of (A.8) and (A.9). For a generic lattice we have:

$$
\mathcal{D}_{y} \mathcal{D}_{x} u_{00}=-\mathcal{D}_{x} \mathcal{D}_{x} u_{00} \frac{\left(h_{00}^{x}-h_{01}^{x}\right)\left(h_{00}^{x}-\sigma_{00}^{x}\right)}{h_{01}^{x} h_{00}^{y}+h_{00}^{x} \sigma_{00}^{y}-h_{01}^{x} \sigma_{00}^{y}-\sigma_{00}^{x} \sigma_{00}^{y}}+
$$


The discretized Boussinesq equation and its conditional symmetry reduction

$$
\begin{aligned}
& +\mathcal{D}_{y} \mathcal{D}_{y} u_{00} \frac{\left(h_{00}^{y}-h_{10}^{y}\right)\left(h_{00}^{y}-\sigma_{00}^{y}\right)}{h_{01}^{x} h_{00}^{y}+h_{00}^{x} \sigma_{00}^{y}-h_{01}^{x} \sigma_{00}^{y}-\sigma_{00}^{x} \sigma_{00}^{y}}+ \\
& +\mathcal{D}_{x} \mathcal{D}_{y} u_{00} \frac{h_{00}^{x} h_{10}^{y}+h_{00}^{y} \sigma_{00}^{x}-h_{1,0}^{y} \sigma_{00}^{x}-\sigma_{00}^{x} \sigma_{00}^{y}}{h_{01}^{x} h_{00}^{y}+h_{00}^{x} \sigma_{00}^{y}-h_{01}^{x} \sigma_{00}^{y}-\sigma_{00}^{x} \sigma_{00}^{y}}
\end{aligned}
$$

Formulas (A.8-A.10) can be simplified if we require the validity of the ClajrautSchwarz-Young theorem i.e. $\mathcal{D}_{x} \mathcal{D}_{y} u_{00}=\mathcal{D}_{y} \mathcal{D}_{x} u_{00}$, i.e.

$$
\begin{aligned}
& \sigma_{n m}^{x}=\sigma_{n+1, m}^{x} \equiv \sigma_{m}^{x}, \quad h_{n m}^{x}=h_{n, m+1}^{x} \equiv h_{n}^{x}, \\
& \sigma_{n m}^{y}=\sigma_{n, m+1}^{y} \equiv \sigma_{n}^{y}, \quad h_{n m}^{y}=h_{n+1, m}^{y} \equiv h_{m}^{y} .
\end{aligned}
$$

Using the operators $\mathcal{D}_{x}$ and $\mathcal{D}_{y}$ given in (A.7) and the new independent variables (A.8) we can transform the standard discrete prolongation

$$
\operatorname{pr} \hat{X}_{n m}=\hat{X}_{n m}+\hat{X}_{n+1, m}+\hat{X}_{n+2, m}+\hat{X}_{n, m+1}+\hat{X}_{n, m+2}+\hat{X}_{n+1, m+1}
$$

of the vector field

$$
\hat{X}_{n m}=\xi_{n m} \partial_{x_{n m}}+\eta_{n m} \partial_{y_{n m}}+\phi_{n m} \partial_{u_{n m}}
$$

to the new set of independent variables (A.8, A.9). We get

$$
\begin{aligned}
& \operatorname{pr} \hat{X}_{n m}=\hat{X}_{n m} \\
& +\sum_{i, j \in\{0,1\}}\left(\zeta_{n+i, m+j}^{(x)} \partial_{h_{n+i, m+j}^{x}}+\chi_{n+i, m+j}^{(x)} \partial_{\sigma_{n+i, m+j}^{x}}+\zeta_{n+i, m+j}^{(y)} \partial_{h_{n+i, m+j}^{y}}+\chi_{n+i, m+j}^{(y)} \partial_{\sigma_{n+i, m+j}^{y}}\right) \\
& \left.+\phi_{n m}^{(1, x)} \partial_{\mathcal{D}_{x} u_{n m}}+\phi_{n m}^{(1, y)} \partial_{\mathcal{D}_{y} u_{n m}}+\phi_{n m}^{(2, x x)} \partial_{\mathcal{D}_{x}^{2} u_{n m}}+\phi_{n m}^{(2, x y)} \partial_{\mathcal{D}_{x} \mathcal{D}_{y} u_{n m}}+\phi_{n m}^{(2, y y)} \partial_{\mathcal{D}_{y}^{2} u_{n m}}, \quad \text { (A. } 14\right)
\end{aligned}
$$

where

$$
\begin{aligned}
\zeta_{n+i, m+j}^{(x)} & =\xi_{n+1+i, m+j}-\xi_{n+i, m+j}, \quad \zeta_{n+i, m+j}^{(y)}=\eta_{n+i, m+1+j}-\eta_{n+i, m+j} \\
\chi_{n+i, m+j}^{(x)} & =\xi_{n+i, m+1+j}-\xi_{n+i, m+j}, \quad \chi_{n+i, m+j}^{(y)}=\eta_{n+1+i, m+j}-\eta_{n+i, m+j} \\
\phi_{n m}^{(1, x)} & =\mathcal{D}_{x} \phi_{n m}-\mathcal{D}_{x} u_{n m} \mathcal{D}_{x} \xi_{n m}-\mathcal{D}_{y} u_{n m} \mathcal{D}_{x} \eta_{n m} \\
\phi_{n m}^{(1, y)} & =\mathcal{D}_{y} \phi_{n m}-\mathcal{D}_{x} u_{n m} \mathcal{D}_{y} \xi_{n m}-\mathcal{D}_{y} u_{n m} \mathcal{D}_{y} \eta_{n m} \\
\phi_{n m}^{(2, x x)} & =\mathcal{D}_{x} \phi_{n m}^{(1, x)}-\mathcal{D}_{x}^{2} u_{n m} \mathcal{D}_{x} \xi_{n m}-\mathcal{D}_{y} \mathcal{D}_{x} u_{n m} \mathcal{D}_{x} \eta_{n m} \\
\phi_{n m}^{(2, x y)} & =\mathcal{D}_{x} \phi_{n m}^{(1, y)}-\mathcal{D}_{x} \mathcal{D}_{y} u_{n m} \mathcal{D}_{x} \xi_{n m}-\mathcal{D}_{y}^{2} u_{n m} \mathcal{D}_{x} \eta_{n m} \\
\phi_{n m}^{(2, y y)} & =\mathcal{D}_{y} \phi_{n m}^{(1, y)}-\mathcal{D}_{x} \mathcal{D}_{y} u_{n m} \mathcal{D}_{y} \xi_{n m}-\mathcal{D}_{y}^{2} u_{n m} \mathcal{D}_{y} \eta_{n m}
\end{aligned}
$$

In the continuous limit, when $h_{n+i, m+j}^{x}, h_{n+i, m+j}^{y}, \sigma_{n+i, m+j}^{x}$ and $\sigma_{n+i, m+j}^{y}$ go to $0, \zeta_{n+i, m+j}^{(x)}$, $\zeta_{n+i, m+j}^{(y)}, \chi_{n+i, m+j}^{(x)}$ and $\chi_{n+i, m+j}^{(y)}$ go also to 0 while $\phi_{n m}^{(1, x)}, \phi_{n m}^{(1, y)}, \phi_{n m}^{(2, x x)}, \phi_{n m}^{(2, x y)}$ and $\phi_{n m}^{(2, y y)}$ go to the corresponding continuous prolongations.

Applying the prolongation (A.14) of the infinitesimal generator $\hat{X}_{n m}$ onto the Schwarzian condition we get that both functions $\xi_{n m}$ and $\eta_{n m}$ must satisfy the discrete wave equations

$$
\begin{aligned}
& \xi_{n, m+1}-\xi_{n m}-\xi_{n+1, m+1}+\xi_{n+1, m}=0, \\
& \eta_{n, m+1}-\eta_{n m}-\eta_{n+1, m+1}+\eta_{n+1, m}=0 .
\end{aligned}
$$

This is a constraint for the symmetry coefficients if the Clairaut-Schwarz-Young theorem is to be satisfied. 
The discretized Boussinesq equation and its conditional symmetry reduction

\section{References}

[1] Aziz T and Mahomed F M 2017 Applications of Group Theoretical Methods to Non-Newtonian Fluid Flow Models: Survey of Results Math. Problems in Eng. 2017

[2] Bihlo A, Coiteux-Roy X and Winternitz P 2015 The Korteweg de Vries equation and its symmetrypreserving discretization J. Phys. A: Math. Theor. 48055201

[3] Bihlo A 2013 Invariant meshless discretization schemes J. Phys. A: Math. Theor. 46062001

[4] Bihlo A and Nave J C 2013 Invariant discretization schemes using evolution-projection techniques SIGMA 9052

[5] Bluman G W and Cole J D 1969 The general similarity solutions of the heat equation J. Math. Mech. 18 1025-1042

[6] Bluman G W and Kumei S 1989 Symmetries and Differential Equations (New York: Springer)

[7] Bourlioux A, Cyr-Gagnon C and Winternitz P 2006 Difference schemes with point symmetries and their numerical tests J. Phys. A $396877-6896$

[8] Boussinesq J 1871 Théorie de l'intumescence liquide appelée onde solitaire ou de translation se propageant dans un canal rectangulaire Comptes Rendus 72 755-759

[9] Boussinesq J 1872 Théorie des ondes et des remous qui se propagent lelong d'un canal rectangulaire horizontal, en communiquant au liquide contenu dans ce canal des vitesses sensiblement pareilles de la surface au fond J. Math. Pures Appl. 7 55-108

[10] Budd C and Dorodnitsyn V A 2001 Symmetry-adapted moving mesh schemes for the nonlinear Schrödinger equation J. Phys. A Math. Theor. 34 10387-10400

[11] Campoamor-Stursberg R, Rodríguez M A and Winternitz P 2016 Symmetry preserving discretization of ordinary differential equations. Large symmetry groups and higher order equations J. Phys. A 49035201

[12] Cherniha R, Serov M and Pliukhin O 2018 Nonlinear Reaction-Diffusion-Convection Equations: Lie and Conditional Symmetry, Exact Solutions and Their Applications (Boca Raton: CRC Press)

[13] Clarkson P A 1995 Nonclassical symmetry reductions of the Boussinesq equation Chaos Solitons Fractals 5 2261-2301

[14] Clarkson P A and Kruskal M D 1989 New similarity reductions of the Boussinesq equation $J$. Math. Phys. 30 2201-2213

[15] Dorodnitsyn V A 1991 Transformation groups in difference spaces J. Soviet Math. 55 1490-1517

[16] Dorodnitsyn V A 2011 Applications of Lie Groups to Difference Equations (Boca Raton: CRC Press)

[17] Dorodnitsyn V A and Kozlov R 2003 A Heat Transfer with a Source: the Complete Set of Invariant Difference Schemes J. Nonl. Math. Phys. 10 16-50

[18] Fushchich W I Shtelen W M and Serov N I 1993 Symmetry Analysis and Exact Solutions of Equations of Nonlinear Mathematical Physics (Dordrecht: Kluwer)

[19] Fushchich W I and Zhdanov R Z 1993 Conditional symmetry and reduction of partial differential equations Ukr. Math. J,44 875-886

[20] Grundland M A and Picard P Y 2004 On Conditionally Invariant Solutions of Magnetohydrodynamic Equations J. Nonl. Math. Phys. 11 47-74

[21] Hydon P E 2014 Difference Equations by Differential Equation Methods (Cambridge: Cambridge University Press)

[22] Ince E L 1956 Ordinary Differential Equations (New York: Dover)

[23] Iserles A 2008 A First Course in the Numerical Analysis of Differential Equations 2nd edition (Cambridge: Cambridge University Press)

[24] Levi/D, Martina L and Winternitz P 2015 Lie-point symmetries of the discrete Liouville equation, J. Phys. A: Math. Theor. 48025204

[25] Levi D, Martina L and Winternitz P 2015 Structure Preserving Discretizations of the Liouville Equation and their Numerical Tests SIGMA 11080 
The discretized Boussinesq equation and its conditional symmetry reduction

[26] Levi D, Martina L and Winternitz P 2018 Conformally invariant elliptic Liouville equation and its symmetry preserving discretization Theor. Math. Phys. 196 1307-1319

[27] Levi D, Olver P J, Thomova Z and Winternitz P (editors) 2011 Symmetries and Integrability of Difference Equations LMS Lecture Series 381 (Cambridge University Press)

[28] Levi D, Ragnisco O and Rodríguez M A 1992 On non-isospectral flows, Painlevé equations, and symmetries of differential and difference equations, Theor. Math. Phys. 93 1409-1414.

[29] Levi D and Rodríguez M A 2013 Construction of partial difference schemes: I. The Clairaut, Schwarz, Young theorem on the lattice J. Phys. A Math. Theor. 46295203

[30] Levi D and Rodríguez M A 2014 On the construction of partial difference schemes II: discrete variables and invariant schemes Acta Polytechnica 56 236-244

[31] Levi D, Rodríguez M A and Thomova Z 2019 Differential Equations Invariant under Conditional Symmetries J. Nonlin. Math. Phys. 26 281-293

[32] Levi D, Rodríguez M A and Thomova Z 2019 Construction of Partial Differential Equations with Conditional Symmetries CRM series in Mathematical Physics - Springer, "Integrability, Supersymmetry and Coherent States. A volume in honor of Professor Véronique Hussin. To be published

[33] Levi D and Winternitz P 1989 Nonclassical symmetry/reduction:/Example of the Boussinesq equation J. Phys. A: Math. Gen. 22 2915-2924

[34] Levi D and Winternitz P 1991 Continuous symmetries of discrete equations Phys. Lett. A 152 335-338

[35] Levi D and Winternitz P 2006 Continuous symmetries of difference equations J. Phys. A: Math. Theor. 39 R1-R63

[36] Levi D, Winternitz P and Yamilov R I 2010 Lie point symmetries of differential-difference equations, J. Phys. A: Math. Theor. 43292002

[37] Levi D, Winternitz P and Yamilov R I 2020 Symmetry and Integrability of Discrete Equations, Chapter on Symmetries as an integrability Criteria, Section on Why Integrable Equations on the Lattice are Symmetrical, AMS, Providence, USA. In preparation

[38] Levi D and Yamilov R I 1995 Classification of evolutionary equations on the lattice. I. The general theory, arXiv preprint solv-int/9511006,

[39] Levi D and Yamilov R I 1997 Conditions for the existence of higher symmetries of evolutionary equations on the lattice, J. Math. Phys. 38 6648-6674

[40] Nishitani T and Tajiri M 1982 On similarity solutions of the Boussinesq equation Phys. Lett. A $89379-380$

[41] Nucci M C and Clarkson P A 1992 The nonclassical method is more general than the direct method for symmetry reductions. An example of the Fitzhugh-Nagumo equation Phys. Lett. A $16449-56$

[42] Olver P J 1993 Applications of Lie groups to differential equations (New York: Springer)

[43] Rebelo R and Valiquette F 2013 Symmetry preserving numerical schemes for partial differential equations and their numerical tests J. Difference Eq. Appl. 19 738-757

[44] Rebelo R and Valiquette F 2015 Invariant discretization of partial differential equations admitting infinite-dimensional symmetry groups J. Difference Eq. Appl. 21 285-318

[45] Rebelo R and Winternitz P 2009 Invariant difference schemes and their applications to SL $(2, \mathrm{R})$ invariant differential equations J. Phys. A. Math. Theor. 42454016

[46] Scott A C 1975 Bäcklund Transformations, Lecture Notes in Mathematics 515 ed. by R. M. Miura, (Berlin: Springer) pp. 80-105.

[47] Scott A C ed. 2005 Encyclopedia of Nonlinear Science Routledge, New York,

[48] Toda M 1975 Studies of a nonlinear lattice Phys. Rep. 18 1-125

[49] Winternitz P 2011 Symmetry preserving discretization of differential equations and Lie point symmetries of differential-difference equations, in Symmetries and Integrability of Difference Equations, London Math. Soc. Lecture Note Ser. Vol. 381, Editors D. Levi, P.J. Olver, Z. Thomova and P. Winternitz, Cambridge University Press, Cambridge, 2011, 292-341. 
[50] Zabusky N J A 1967 Synergetic Approach to Problems of Nonlinear Dispersive Wave Propagation and Interaction, in Nonlinear Partial Differential Equations ed. by W.F. Ames, (New York: Academic Press) pp. 233-258

[51] Zakharov V E 1974 On stochastization of one-dimensional chains of nonlinear oscillations Sov. Phys. JETP 38 108-110

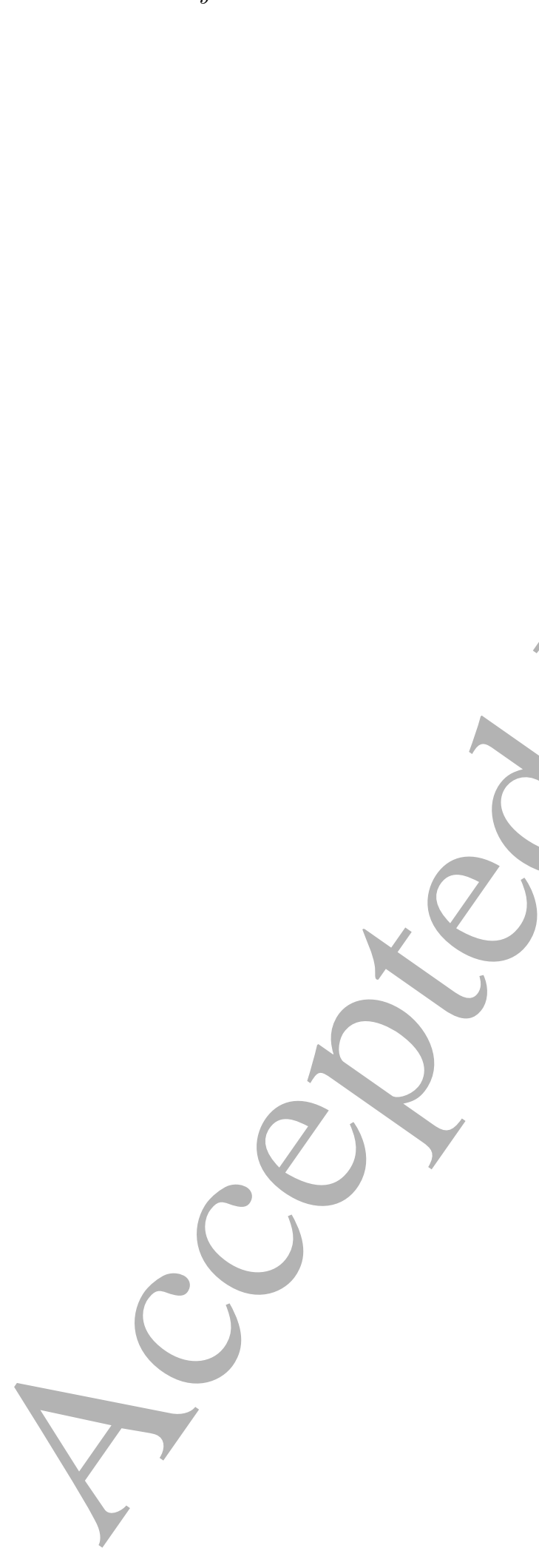

